\title{
Recent Advances in Highly Nonlinear Fibres
}

\author{
D.J. Richardson ${ }^{1}$, X. Feng ${ }^{1}$, F. Poletti ${ }^{1}$, S. Dasgupta ${ }^{1}$ A. Camerlingo ${ }^{1}$, F. Parmigiani ${ }^{1}$, P. Petropoulos ${ }^{1}$, \\ W. H. Loh ${ }^{1}$, Søren Herstrøm ${ }^{2}$ and Lars Grüner-Nielsen ${ }^{2}$ \\ (1) Optoelectronics Research Centre, University of Southampton, United Kingdom, djr@orc.soton.ac.uk \\ (2) OFS Denmark, Priorparken 680, DK-2605 Brøndby, Denmark, Igruner@ofsoptics.com
}

\begin{abstract}
We review recent progress in the areas of silica and compound glass based highly nonlinear fibres, highlighting and contrasting the current start-of-the-art, merits, drawbacks and future potential of both approaches.
\end{abstract}

\section{Introduction}

The ability to manipulate and process signals directly within the optical domain is highly desirable in many areas of photonics. Arguably this is most important in the context of telecommunications where the possibility to perform functions such as broadband wavelength conversion, ultrafast all-optical routing and regeneration, offer significant benefits to system operators providing robust, compact and reliable devices can be realised. Critical to the development of reliable nonlinear optical devices is the choice of nonlinear medium: both with respect to the nature of the nonlinear effects that can be exploited (in terms of speed-of-response, bandwidth, cross-talk etc.), and practicality (in terms of power requirements, size and ease of fibre interconnection etc.).

Whilst semiconductor devices (both active and passive), chalcogenide planar waveguides and periodically-poled crystalline based components remain major contenders, the majority of work on telecomm based nonlinear processing to date has focused on exploiting Kerr-based effects in highly nonlinear optical fibres (HNLFs) [1]. Tremendous advances have been made in recent years in HNLF fabrication technology using both the more conventional and well-established silica-based materials and more recently using compound glasses which offer substantially higher values of material nonlinearity (albeit with substantially higher losses). Generally speaking, the primary benefits of a fibre approach relate to: the ultrafast response times of glasses $(<5 \mathrm{fs})$; the very low propagation losses that can be achieved (e.g. $<1 \mathrm{~dB} / \mathrm{km}$ for silica based HNLFs that lead to a very high figure-of-merit $\left(n_{2} / \alpha\right)$ and practical power requirements for device lengths of order $100 \mathrm{~m}$ ); the level of dispersion control that can be achieved and used to engineer the overall nonlinear response of the fibre; and the ready integration into fibre systems. Despite these benefits there are a number of technical issues associated with fibre based approaches that need to be considered - firstly it is still necessary to use relatively long device lengths leading to potential issues with latency and environmental sensitivity; accurate dispersion control is usually required which is extremely challenging from a fibre manufacturing perspective; Kerr based effects are polarisation sensitive meaning that the fabrication of fibres with well defined polarisation properties becomes important; and finally Stimulated Brillouin Scattering (SBS) can become a very significant limiting factor for applications requiring narrow-linewidth signals.

Within this paper we provide a brief overview of recent progress in the areas of HNLF development, considering separately both silica-based and compound glass-based variants that address several of the key issues outlined above. Our objective is not to give a full and complete review of the field but to give some insight into the relative state of development of both approaches and to allow a comparative assessment of their future potential and key challenges that lie ahead.

\section{Solid silica HNLFs}

In Table 1 we show the typical properties of three types of HNLF developed by OFS: "Standard-HNLF" with a dispersion-slope of $0.019 \mathrm{ps} /\left(\mathrm{nm}^{2} \cdot \mathrm{km}\right)$, dispersion-flattened HNLF [2], and a recently developed Polarisation-Maintaining HNLF based on an elliptical core [3].

\begin{tabular}{|c|c|c|c|c|}
\hline & & Standard & Flattened & PM \\
\hline Effective area & $\mu \mathrm{m}^{2}$ & 11.6 & 12.4 & 12.5 \\
\hline $\begin{array}{c}\text { Nonlinear } \\
\text { coefficient }(\mathrm{y})\end{array}$ & $W^{-1} \mathrm{~km}^{-1}$ & 11.5 & 10.8 & 10.7 \\
\hline Dispersion & $\mathrm{ps} /(\mathrm{nm} \cdot \mathrm{km})$ & Selected & Selected & Selected \\
\hline $\begin{array}{c}\text { Dispersion } \\
\text { slope }\end{array}$ & $\mathrm{ps} /\left(\mathrm{nm}^{2} \cdot \mathrm{km}\right)$ & 0.019 & 0.007 & 0.025 \\
\hline Attenuation & $\mathrm{dB} / \mathrm{km}$ & 0.8 & 0.8 & 0.8 \\
\hline $\begin{array}{l}\text { Splice loss to } \\
\text { standard fibre }\end{array}$ & $\mathrm{dB}$ & 0.1 & 0.1 & 0.3 \\
\hline $\begin{array}{c}\text { Polarization } \\
\text { extinction }\end{array}$ & $\mathrm{dB}$ & & & 24 \\
\hline Birefringence & & & & $3 \cdot 10^{-4}$ \\
\hline $\begin{array}{c}\text { Cut-off } \\
\text { wavelength }\end{array}$ & $\mathrm{nm}$ & 1180 & 1240 & 1300 \\
\hline
\end{tabular}

Table 1. Typical properties of various OFS HNLFs as measured at $1550 \mathrm{~nm}$.

For each type of fibre the dispersion can be substantially tailored to suit the end-application. For example, parametric amplification requires a zero 
dispersion wavelength close to the pump, supercontinuum generation requires a moderate anomalous dispersion coefficient, and pulse compression typically requires a moderate normal dispersion coefficient. In Figure 1 we show some specific examples of measured dispersion profiles for the various fibre types described in Table 1.

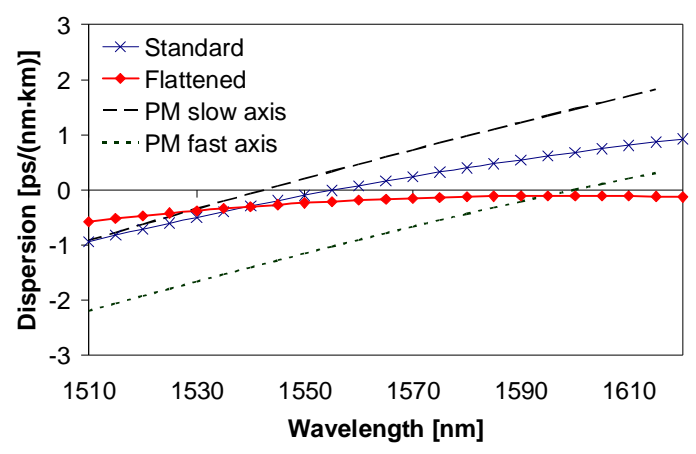

Figure 1. Examples of typical dispersion profiles (as a function of wavelength) for various OFS HNLFs.

As previously mentioned, SBS limits the signal power that can be launched into the HNLF and is thus, a major issue. Several "in-fibre" methods for SBS suppression have been proposed that broaden the effective gain-bandwidth and increase the SBS threshold. These include applying a linear or stepwise temperature-gradient along the HNLF [4] or inducing a linear or stepwise strain-gradient along the fibre [5].

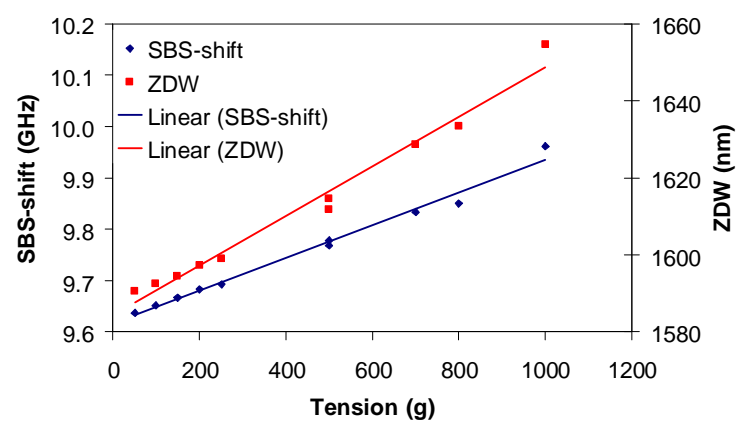

Figure 2. Measured change in SBS frequency shift and zero dispersion wavelength versus spooling tension. Also shown are linear fits to the measured data.

SBS suppression by straining the HNLF is obtained since the SBS gain peak is shifted in wavelength when the fibre is strained. So, by spooling the HNLF with a linear strain-gradient along its length the SBS gain spectrum can be broadened and the SBS threshold consequently increased. A drawback of the tensioning is that the tension also changes the dispersion properties of the HNLF. In Figure 2 we show an example of the measured effect of spool tension on both SBS shift and dispersion for a standard HNLF. Using this approach a $7 \mathrm{~dB}$ increase in SBS threshold has been measured for standard HNLF with linearly-ramped tension from $1000 \mathrm{~g}$ to $40 \mathrm{~g}$ along its $300 \mathrm{~m}$ length.
An alternative passive approach to SBS suppression is to adopt special fibre designs that modify the acoustic guidance properties of the fibre by doping the core with aluminium $[6,7]$ such that the core becomes an anti-guide for the acoustic waves responsible for SBS. This reduces the interaction between the optical and acoustic waves and provides for significant increases in SBS thresholds - e.g. by $6 \mathrm{~dB}$ in Reference [7].

\section{Compound Glass HNLFs}

Whilst silica-based fibres provide excellent performance hundreds metre lengths are needed for most applications due to the low Kerr index of silica. Compound glass materials offer anything up to a thousand-fold increase in Kerr index and thus offer the potential for $<1 \mathrm{~m}$ devices operating at $\mathrm{mW}$ power levels. Moreover, it is to be appreciated that the relative strength of the Kerr to Brillouin nonlinearities in many compound glasses is much greater than in silica and consequently SBS is often much less of an issue than for silica HNLFs. A number of compound glass fibres have been reported over the years with arguably most success achieved with Bismuth based glasses with $y$ values well in excess of $1000\left(\mathrm{Wkm}^{-1}\right.$ at $1550 \mathrm{~nm}$ demonstrated for step-index fibres [8]. However, with the advent of microstructured optical fibre (MOF) technology, it has now become far simpler to make fibres in compound glass materials since no additional optically and thermally compatible cladding material is required. Furthermore, the technology allows for excellent broadband dispersion control and ultrahigh effective numerical apertures for certain designs - such as the Air Suspended Core (ASC) structure shown in Fig. 3a. The high-NA allows for ultra small-cores and hence extremely high values of $y$ [9]. (Note that in the sub-wavelength core regime the longitudinal field component become significant which can further enhance the nonlinearity as shown in [10]). Using such an approach it has been possible to obtain values of gamma as high as 5400 (W.km) ${ }^{-1}$ in a bismuth glass fibre [10] and in excess of 15,000 (W.km) $)^{-1}$ in a chalcogenide fibre [11]. By pushing to the very minimum core dimensions and using the most nonlinear glasses $\mathrm{y}$ values approaching 100,000 (W.km) $)^{-1}$ should be possible.

Whilst pushing to such extremes of nonlinearity is extremely interesting, it is important to note that as the effective area is reduced device losses increase due to the increased sensitivity to surface roughness, coupling losses to standard fibres increase rapidly and the values of dispersion around $1550 \mathrm{~nm}$ tend to be very high. Consequently, for many telecoms applications such fibres are non-optimal when the full device performance is considered. In these instances more complex structures are required in which maximum nonlinearity is traded off for improved dispersion properties, or lower overall device loss. An 
example of a dispersion-shifted fibre design produced in SF57 lead-silicate glass is shown in Figure 3b. In this fibre $y$ has been reduced to around $15 \%$ of the maximum possible value in order to achieve the desired dispersion-shifted performance. It is however to be appreciated that achieving the necessary dispersion accuracy is extremely challenging due to deformation of the air holes during the MOF fibre drawing process (indeed the final geometry is only really defined in this final fabrication step).
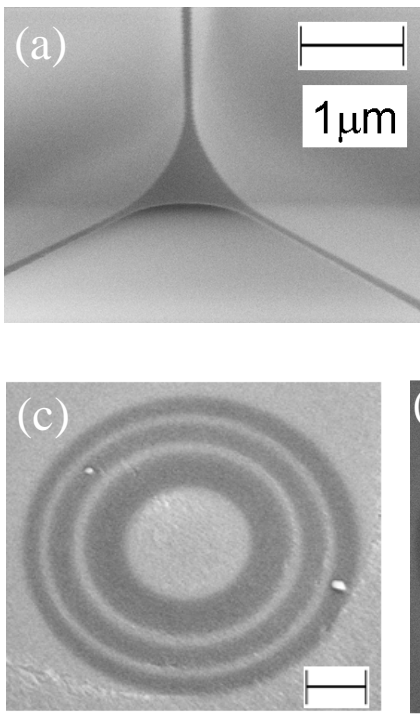

$2 \mu \mathrm{m}$

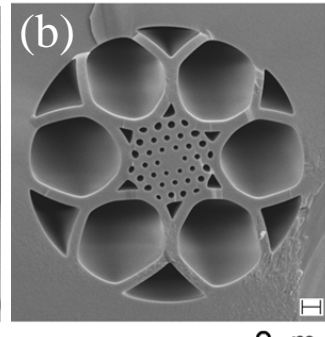

$2 \mu \mathrm{m}$
SF57

Figure 3. (a) Air Suspended Core ultrahigh $\gamma$ fibre in SF57 glass, (b) dispersion-shifted SF57 MOF, (c) solid 1-D dispersion-shifted MOF and (d) dispersion-flattened W-fibre.

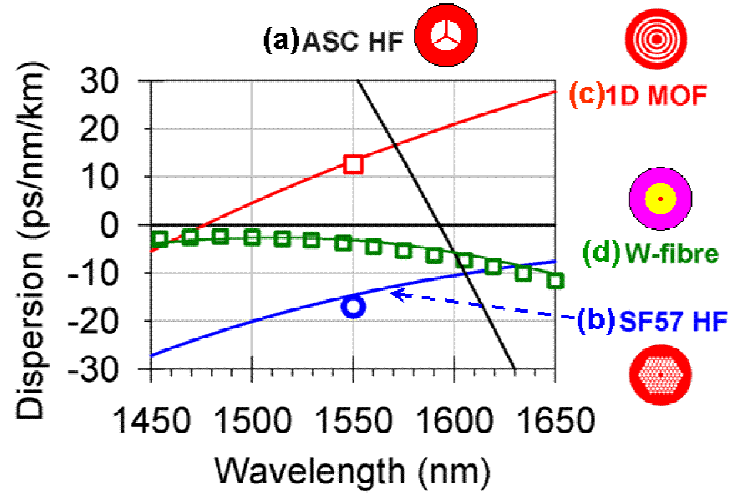

Figure 4. The dispersion characteristics of the various fibres shown above. The symbols represent measured spot values. The associated y values are 1850, 280, 120 and 820 $(W . k m)^{-1}$ for fibres (a) to (d) respectively.

In order to improve the fabrication accuracy it is preferable to move to all-solid MOF designs using different combinations of sufficiently thermally and optically matched glasses. In this instance the geometric refractive index structure is defined at the preform stage and fibre drawing is purely for scale reduction [12]. Different approaches can be used to produce the solid preforms including stacking and extrusion and two or more glass types might be included within the structure. Two examples, a Bragg type fibre [12], and a W-type structure, are presented in Figure 4 [13].

The fibre in Figure $3 \mathrm{c}$ was fabricated using highly polished disks extruded through a circular aperture and represents a dispersion-shifted HNLF design. This particular fibre is characterised by an extremely low loss of $0.8 \mathrm{~dB} / \mathrm{m}$ which is comparable to that of the bulk glass used for the core. The fibre shown in Figure $3 d$ is a W-type fibre offering a flat low dispersion at $1550 \mathrm{~nm}$ (see Figure 4 for a summary of the dispersion characteristics of the various fibres shown in Figure 3 ). The measured nonlinearity of this fibre is $820(\mathrm{~W} . \mathrm{km})^{-1}$ - a record value for a dispersionflattened HNLF at $1550 \mathrm{~nm}$. The current loss value is $\sim 2 \mathrm{~dB} / \mathrm{m}$, although further reduction to the materialloss level of $1 \mathrm{~dB} / \mathrm{m}$ is fully expected in due course, with further reduction possible using improved purity glass. Using higher index glass types it will be possible to scale the nonlinearity substantially further for this and indeed all of the other MOFs described in this paper. Note that as well as allowing greater fabrication accuracy the use of solid MOF structures greatly simplifies the splicing of these exotic fibre types to external silica fibres.

\section{Conclusion}

In conclusion, we have reviewed recent advances in HNLF research which illustrate the high performance levels now possible in silica based variants and the prospects for dispersion-managed ultrahigh nonlinearity compound glass devices.

This research was funded in part by the European Communities Seventh Framework Programme FP/2007-2013 (224547, PHASORS).

\section{References}

[1] M. J. Holmes et al., IEEE J. Lightwave Tech. 7, 1045 (1995).

[2] C. Jørgensen et al., Proc. ECOC'03, We3.7.6 (2003

[3] L Grüner-Nielsen et al., Proc. ECOC'08, Mo4.B.6 (2008).

[4] J. Hansryd et al., IEEE J. Lightwave Tech. 19, 1691 (2002).

[5] J. M. Chavez Boggio et al., IEEE J. Lightwave Tech. 23, 3808 (2005).

[6] T. Nakasinishi et al., Proc. ECOC'06, Th4.2.2 (2006).

[7] L. Grüner-Nielsen et al., Proc. ECOC'10 (2010).

[8] K. Kikuchi et al., Electron. Letts., 38, 166-167, (2002)

[9] J.Y.Y. Leong et al., IEEE J. Lightwave Tech., 24(1) 183-190, (2006).

[10] T.M. Monro et al., IEEE J. Quantum. Electron., 45, 1357-1364, (2009).

[11] T.N. Nguyen et al., Proc. ECOC 2009, (2009).

[12] X. Feng et al., Opt. Express, 17(22), 20249, (2009).

[13] X. Feng et al. Proceedings of ECOC'10 (2010). 